\title{
Application of the uniformized Mittag-Leffler expansion to $\Lambda(1405)$
}

\author{
Wren A. Yamada ${ }^{1,2, *}$ and Osamu Morimatsu ${ }^{10}{ }^{1,2,3, \dagger}$ \\ ${ }^{1}$ Department of Physics, Faculty of Science, University of Tokyo, 7-3-1 Hongo Bunkyo-ku Tokyo 113-0033, Japan \\ ${ }^{2}$ Theory Center, Institute of Particle and Nuclear Studies (IPNS), High Energy Accelerator Research Organization (KEK), \\ 1-1 Oho, Tsukuba, Ibaraki, 205-0801, Japan \\ ${ }^{3}$ Department of Particle and Nuclear Studies, Graduate University for Advanced Studies (SOKENDAI), 1-1 Oho, \\ Tsukuba, Ibaraki 305-0801, Japan
}

(Received 30 January 2021; accepted 2 March 2021; published 1 April 2021)

\begin{abstract}
We study the pole properties of $\Lambda(1405)$ in a model-independent manner by applying the uniformized MittagLeffler expansion proposed in our previous paper. The resonant energy, width and residues are determined by expanding the observable as a sum of resonant-pole pairs under an appropriate parametrization which expresses the observable to be single valued, and fitting it to experimental data of the invariant-mass distribution of $\pi^{+} \Sigma^{-}$, $\pi^{-} \Sigma^{+}, \pi^{0} \Sigma^{0}$ final states in the reaction $\gamma p \rightarrow K^{+} \pi \Sigma$ and the elastic and inelastic cross section $K^{-} p \rightarrow K^{-} p$, $\bar{K}^{0} n, \pi^{+} \Sigma^{-}, \pi^{-} \Sigma^{+}$. As we gradually increase the number of pairs from one to three, the first pair converges while the second and third pairs emerge further and further away from the first pair, implying that the uniformized Mittag-Leffler expansion with three pairs is almost convergent in the vicinity of the $\Lambda(1405)$. The broad peak structure between the $\pi \Sigma$ and $\bar{K} N$ thresholds regarded to be $\Lambda(1405)$ is explained by a single pair with a resonant energy of $1420 \pm 1 \mathrm{MeV}$, and a half- width of $48 \pm 2 \mathrm{MeV}$, which is consistent with the single-pole picture of $\Lambda$ (1405). We conclude that the uniformized Mittag-Leffler expansion turns out to be a very powerful and simple method to obtain resonance energy, width, and residues from the near-threshold spectrum.
\end{abstract}

DOI: 10.1103/PhysRevC.103.045201

\section{INTRODUCTION}

In recent years many hadron resonances, in particular, candidates of exotic hadrons have been found near the thresholds of hadronic channels [1,2]. Due to the threshold effects, their spectra are significantly distorted from the Breit-Wigner form [3],

$$
\mathcal{A}(\sqrt{s}) \propto \frac{\Gamma_{R}}{\sqrt{s}-M_{R}-i \Gamma_{R} / 2},
$$

making it challenging to extract information of resonances such as the resonance energy and width from the observed spectra in a model-independent manner.

In our previous paper [4], we proposed the uniformized Mittag-Leffler expansion approach, a model-independent approach that incorporates the resonant and threshold behavior appropriately. We showed that when choosing an appropriate parametrization [5,6], the $S$ matrix is a meromorphic function and can be expressed by the Mittag-Leffler expansion [7-10],

\footnotetext{
*wren-phys@g.ecc.u-tokyo.ac.jp

†osamu.morimatsu@kek.jp
}

Published by the American Physical Society under the terms of the Creative Commons Attribution 4.0 International license. Further distribution of this work must maintain attribution to the author(s) and the published article's title, journal citation, and DOI. Funded by $S C O A P^{3}$. as shown in Fig. 1. It is explicitly written by the positions and residues of the bound and resonant poles. The symmetry condition of the pole properties of the $S$ matrix forces the series to obey the proper threshold behavior. Following our proposition, we demonstrated the method by using data of a doublechannel model calculation, with isospin $I=0, \bar{K} N$, and $\pi \Sigma$ channels.

The next step would naturally be the demonstration of the method to actual hadronic spectra. In the present paper, we apply the uniformized Mittag-Leffler expansion to experimental data of the spectrum around $\Lambda(1405)$; a resonance situated between the $\pi \Sigma$ and $\bar{K} N$ threshold [11,12], which has been a topic of interest in studies involving baryons with strangeness [13-15]. It has been naturally described as a hadronic molecular state generated from hadronic degrees of freedom [1], while hardly interpreted as an excitation in the standard threequark description. Moreover, calculations in the chiral-unitary model, such as [16-21], display a double-pole structure in the region of $\Lambda(1405)$, contrary to phenomenological local potential models, such as in Refs. [22-25], which predict a single-pole structure. To settle the debate between the single-pole or double-pole structure of $\Lambda(1405)$, a modelindependent analysis is strongly in need. Owing to these circumstances, the $\Lambda(1405)$ resonance serves as an ideal target for the application of our method.

We apply the uniformized Mittag-Leffler expansion approach to the scattering processes of $K^{-} p$ elastic and inelastic cross sections [26-32], and the invariant-mass distributions of $\pi \Sigma$ final states in the reaction, $\gamma p \rightarrow K^{+} \pi \Sigma$, measured 


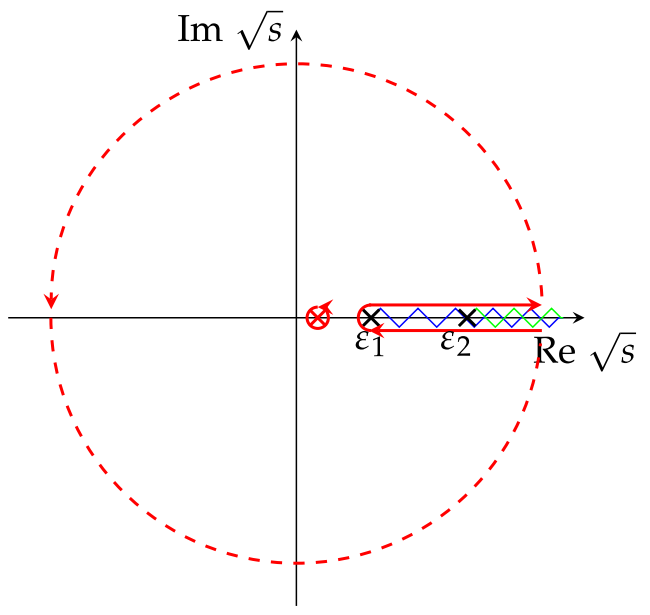

(a)

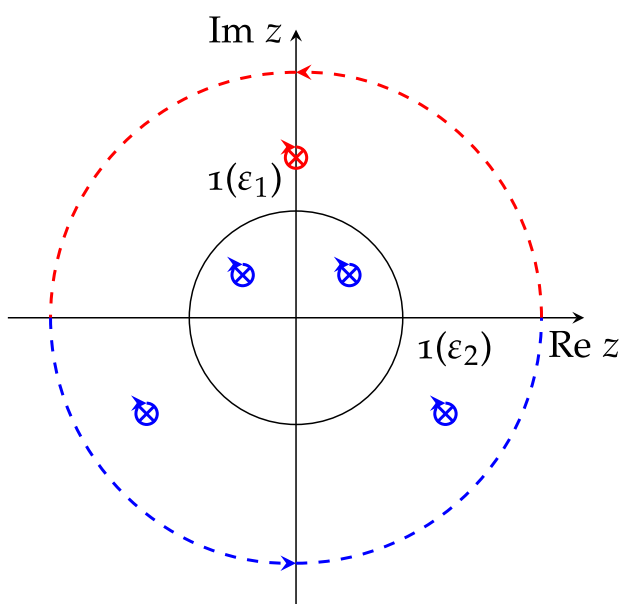

(b)

FIG. 1. (a) Schematic figure of the spectral representation, and the uniformized Mittag-Leffler expansion, of the $\mathcal{S}$ matrix in the case of a double-channel two-body system. $\epsilon_{1}, \epsilon_{2}$ are the threshold energies of the two channels. The continuum contribution along the unitary cuts in panel (a), can be decomposed into resonant contributions from poles in the unphysical domain (blue) in panel (b).

with CLAS at Jefferson Lab [33]. Under the assumption that the spectrum is dominantly generated from the coupledchannel dynamics of the $\pi \Sigma-\bar{K} N$ system, we determine the resonance energy, width, and residues of $\Lambda(1405)$ in a modelindependent manner.

\section{UNIFORMIZED MITTAG-LEFFLER EXPANSION APPROACH}

Here we will briefly review our approach proposed in Ref. [4] for a better understanding of its application to actual experimental data in the following section and to clarify our conventions.

From the Cauchy integration principle, a meromorphic function $f(z)$ can be written as

$$
f(z)=\frac{1}{2 \pi i} \oint_{\gamma} d w \frac{f(w)}{w-z}+\sum_{n} \frac{c_{n}}{z-z_{n}},
$$

where $z_{n}, c_{n}$ are the poles and residues of $f$, and $\gamma$ is a circular contour around the origin with a radius taken to infinity. If the integral term in Eq. (2) vanishes as we take the radius of $\gamma$ to infinity, the meromorphic function $f$ can be written as

$$
f(z)=\sum_{n} \frac{c_{n}}{z-z_{n}},
$$

which is a Mittag-Leffler expansion of $f$. Note that this form is explicitly written by a simple series of the pole position and the residue of $f$.

When the integral term in Eq. (2) does not vanish, or diverges, we can always consider a subtraction, so that the integral takes a form with better convergence to zero [34]. For example, let us consider $g(z)=[f(z)-f(0)] / z$ instead of $f(z)$ in Eq. (2). If the integral term in Eq. (2) for $g(z)$ vanishes, the once-subtracted form of Eq. (3) can be written as

$$
f(z)=f(0)+\sum_{n}\left[\frac{c_{n}}{z-z_{n}}+\frac{c_{n}}{z_{n}}\right],
$$

which differs from Eq. (3) by a constant that corresponds to the subtraction.

Now, let us consider a two-body system. Observables, such as two-body cross sections, $\sigma$, or the distributions of two-body final states with invariant mass $M$, in some reactions (e.g., $\pi \Sigma$ final states in the reaction, $\gamma p \rightarrow K^{+} \pi \Sigma$, schematically shown in Fig. 2), $d \sigma / d M$ are related to the $T$ matrix $\mathcal{T}$ and Green's function $\mathcal{G}$ as [35-37]

$$
\sigma=\frac{1}{16 \pi^{2} s} \frac{k_{f}}{k_{i}} \operatorname{Im} \mathcal{T}
$$

or

$$
\frac{d \sigma}{d M}=\operatorname{Im} \mathcal{F}^{\dagger} \mathcal{G} \mathcal{F}
$$

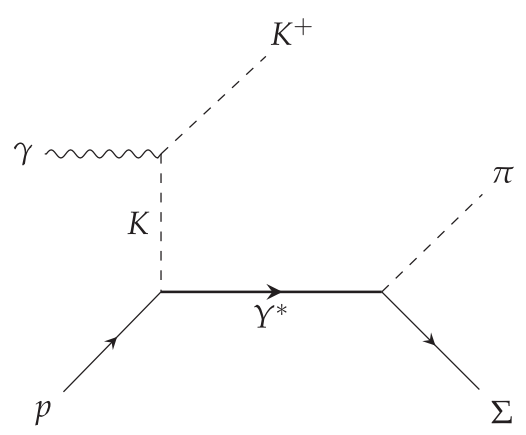

FIG. 2. A schematic diagram of the process ' $\gamma p \rightarrow K^{+} Y^{*} \rightarrow$ $K^{+} \pi \Sigma^{\prime}$ measured in the CLAS experiment [33]. 
(a)

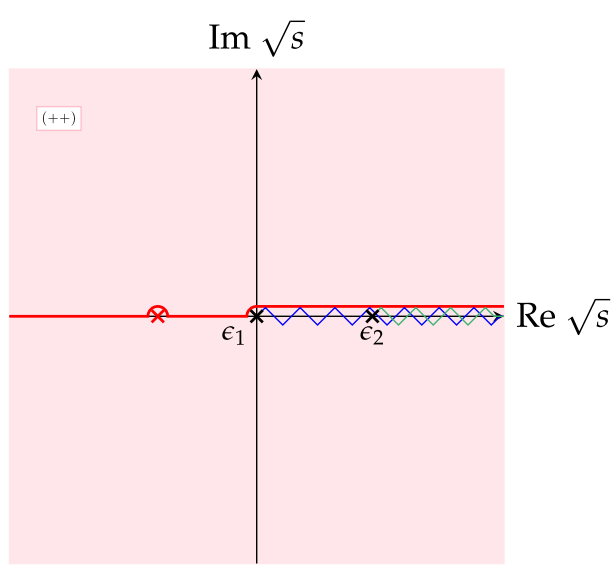

(b)

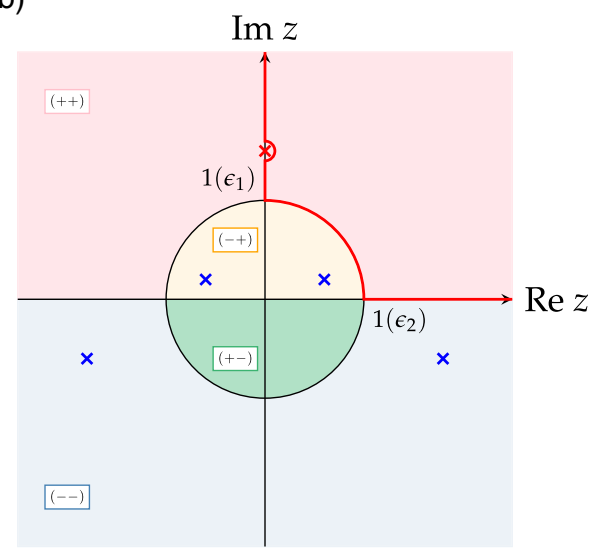

FIG. 3. (a) Analytic structure of the double-channel $\mathcal{S}$ matrix in the $\sqrt{s}$ plane, and (b) the uniformized $z$ plane. In the $\sqrt{s}$ plane, the unitary cuts run along the real axis from $\epsilon_{1}$ to $\infty$ (blue) and from $\epsilon_{2}$ to $\infty$ (green), and the four Riemann sheets of the $\sqrt{s}$ plane correspond to each region in $z$ labeled as $( \pm \pm)$. The red line shows the physical region accessible by experiment.

where $s$ is the center-of-mass energy squared, $k_{f}, k_{i}$ are the final and initial momenta in the center-of-mass frame, respectively, and $\mathcal{F}$ represents the vertex producing two-body final states. For our convenience let $\mathcal{A}$ represent either $\mathcal{T}$ or $\mathcal{F} \dagger \mathcal{G F}$. $\mathcal{A}$ has the same analytic structure as the $S$ matrix.

From the unitary condition, the $S$ matrix has a branch cut running from each threshold along the positive real axis in the $\sqrt{s}$ plane to infinity, known as unitary cuts. Thus, in the variable $\sqrt{s}, \mathcal{A}$ is not meromorphic, and Eq. (3) or (4) cannot be applied directly. To explicitly write $\mathcal{A}$ in the form of a Mittag-Leffler expansion, one must choose an appropriate parametrization so that $\mathcal{A}$ becomes meromorphic. This process is called uniformization [5]. Once uniformization is performed, $\mathcal{A}$ can be decomposed into a series of the form of Eq. (3) or (4). In addition, the unitarity of the $S$ matrix also imposes a symmetry condition on the position of the pole positions and the residues of $\mathcal{A}$. The poles are positioned symmetric about the imaginary axis in the uniformized $z$ plane, and the residues $c_{n}\left(z_{n}\right)$ satisfy the following relationship:

$$
c_{n}\left(z_{n}\right)=-c_{n}^{*}\left(-z_{n}^{*}\right) .
$$

Note that, when considering the pole symmetry condition, the subtraction constant in Eq. (4) is real, and thus the imaginary part of Eqs. (3) and (4) take the same form.

To summarize, by an appropriate choice of variable $z$, the imaginary part of $\mathcal{A}$ can be written as

$$
\operatorname{Im} \mathcal{A}(z)=\operatorname{Im} \sum_{n}\left(\frac{c_{n}}{z-z_{n}}-\frac{c_{n}^{*}}{z+z_{n}^{*}}\right),
$$

which we will call the uniformized Mittag-Leffler expansion. Expressing observables in the form of the uniformized Mittag-Leffler expansion and comparing them with the actual experimental data, we can obtain the pole positions and residues of the observables from experimental data in a model-independent manner. Let us call this the uniformized Mittag-Leffler expansion approach.

Explicit procedures are as follows:

(i) Find an appropriate kinetic variable $z$ which uniformizes the $\mathcal{S}$ matrix.

(ii) Truncate the uniformized Mittag-Leffler expansion and approximate $\mathcal{A}(z)$ by a few $(m)$ pairs of the pole terms as

$$
\operatorname{Im} \mathcal{A}(z)=\operatorname{Im} \sum_{n=1}^{m}\left(\frac{c_{n}^{(m)}}{z-z_{n}^{(m)}}-\frac{c_{n}^{(m) *}}{z+z_{n}^{(m) *}}\right) .
$$

(iii) Determine the complex pole positions $z_{n}^{(m)}$ and residues $c_{n}^{(m)}(n=1, \ldots, m)$ by fitting $\mathcal{A}$ to the experimental data.

iv Regard converged $z_{n}^{(m)}, c_{n}^{(m)}$ as the actual pole positions and residues.

The two-body double-channel $\mathcal{S}$ matrix can be expressed as a four-sheeted Riemann surface with unitary cuts running from each threshold $\epsilon_{1}, \epsilon_{2}$ to $\infty$ along the real axis, in the parametrization of the center-of-mass energy $\sqrt{s}$. The threshold energy $\epsilon_{i}$ is

$$
\epsilon_{i}=M_{i}+m_{i},
$$

TABLE I. Results for the pole positions by the uniformized Mittag-Leffler expansion with $m=3 . z_{n}$ is the dimensionless pole position on the $z$ plane and $\sqrt{s}_{n}$ in units of $\mathrm{GeV}$ on the $\sqrt{s}$ plane.

\begin{tabular}{llll}
\hline \hline & Pole 1 & Pole 2 & Pole 3 \\
\hline$z_{n}^{(3)}$ & $0.5243+0.3159 i \pm 0.0062 \pm 0.0058 i$ & $1.6402-1.042 i \pm 0.0684 \pm 0.0904 i$ & $2.3227-0.0687 i \pm 0.0033 \pm 0.0031 i$ \\
$\sqrt{s_{n}^{(3)}}$ & $1.4203-0.0475 i \pm 0.0011 \pm 0.0015 i$ & $1.4283-0.074 i \pm 0.01 \pm 0.0037 i$ & $1.5138-0.0068 i \pm 0.0003 \pm 0.0003 i$ \\
\hline \hline
\end{tabular}




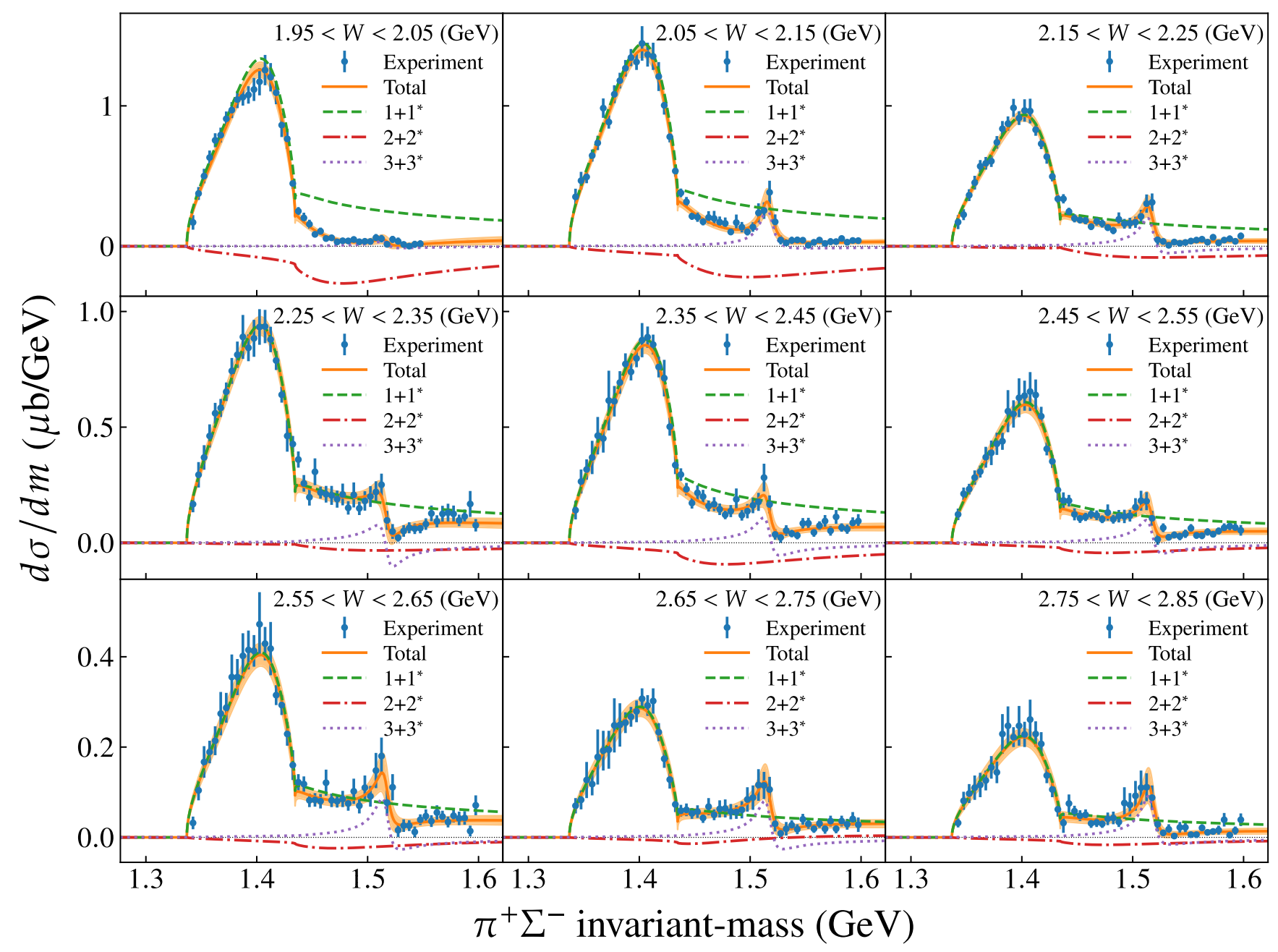

FIG. 4. Results for the invariant-mass distributions of $\pi^{+} \Sigma^{-}$in the reaction $\gamma p \rightarrow K^{+} \pi \Sigma$ in nine bins of center-of-mass energy $W$. The (blue) dots with bars are the experimental data. The (orange) bands represent the $2 \sigma$-confidence interval of the fit by the uniformized MittagLeffler expansion with $m=3$. The (green) dashed, (red) dot-dashed, and (purple) dotted lines represent the contributions from individual resonant-pole pairs of $1+1^{*}, 2+2^{*}$, and $3+3^{*}$, respectively.

where $M_{i}$ and $m_{i}$ are the masses of the two particles in channels $i=1$ and 2 , respectively. The four sheets in the $\sqrt{s}$ plane can be uniquely labeled by the sign of the imaginary part of $q_{1}$ and $q_{2}$, given by

$$
q_{i}=\sqrt{s-\epsilon_{i}^{2}}
$$

which has a one-to-one correspondence with channel momenta. In this paper we label the four sheets by $\left(\sigma\left(q_{1}\right) \sigma\left(q_{2}\right)\right)$ where

$$
\sigma\left(q_{i}\right)=\operatorname{sgn}\left(\operatorname{Im} q_{i}\right)
$$

The physical sheet corresponds to sheet $(++)$.

By the parametrization $z$ [6],

$$
z=\frac{1+\sqrt{u}}{1-\sqrt{u}}
$$

where

$$
u=\frac{q_{1}-\Delta}{q_{1}+\Delta} \quad \text { and } \quad \Delta=\sqrt{\epsilon_{2}^{2}-\epsilon_{1}^{2}}
$$

the four-sheeted Riemann surface can be uniformized into a single complex plane so that $\mathcal{S}(z)$ is meromorphic. The correspondence between the $\sqrt{s}$ plane and $z$ plane is shown in Fig. 3. The two thresholds, $\sqrt{s}=\epsilon_{1}$ and $\sqrt{s}=\epsilon_{2}$ are transformed to points on the unit circle $z=i$ and $z=1$, respectively. The imaginary axis above $i$, the unit circle between $i$ and 1 , and the real axis above 1 correspond to the physically accessible region of $\sqrt{s}<\epsilon_{1}, \epsilon_{1}<\sqrt{s}<\epsilon_{2}$ and $\sqrt{s}<\epsilon_{2}$, respectively.

The contribution of a single resonant-pole pair $\mathcal{A}_{n}$ is given in the vicinity of $\sqrt{s}=\epsilon_{1}$ as

$$
\operatorname{Im} \mathcal{A}_{n}(z)= \begin{cases}0 & \left(\sqrt{s}<\epsilon_{1}\right) \\ -\operatorname{Im} \frac{2 c_{n}}{\left(z_{n}-i\right)^{2}} \frac{k_{1}}{\Delta}+O\left(k_{1}^{2}\right) & \left(\sqrt{s}>\epsilon_{1}\right),\end{cases}
$$

and in the vicinity of $\sqrt{s}=\epsilon_{2}$ as

$$
\operatorname{Im} \mathcal{A}_{n}(z)= \begin{cases}\operatorname{Im} \frac{2 c_{n}}{1-z_{n}^{2}}-\operatorname{Re} \frac{4 c_{n} z_{n}}{\left(1-z_{n}^{2}\right.} \tilde{k}_{2}^{2}+O\left(\tilde{k}_{2}^{2}\right) & \left(\sqrt{s}<\epsilon_{2}\right) \\ \operatorname{Im} \frac{2 c_{n}}{1-z_{n}^{2}}-\operatorname{Im} \frac{2 c_{n}\left(1+z_{n}^{2}\right)}{\left(1-z_{n}^{2}\right)^{2}} \frac{k_{2}}{\Delta}+O\left(k_{2}^{2}\right) & \left(\sqrt{s}>\epsilon_{2}\right),\end{cases}
$$




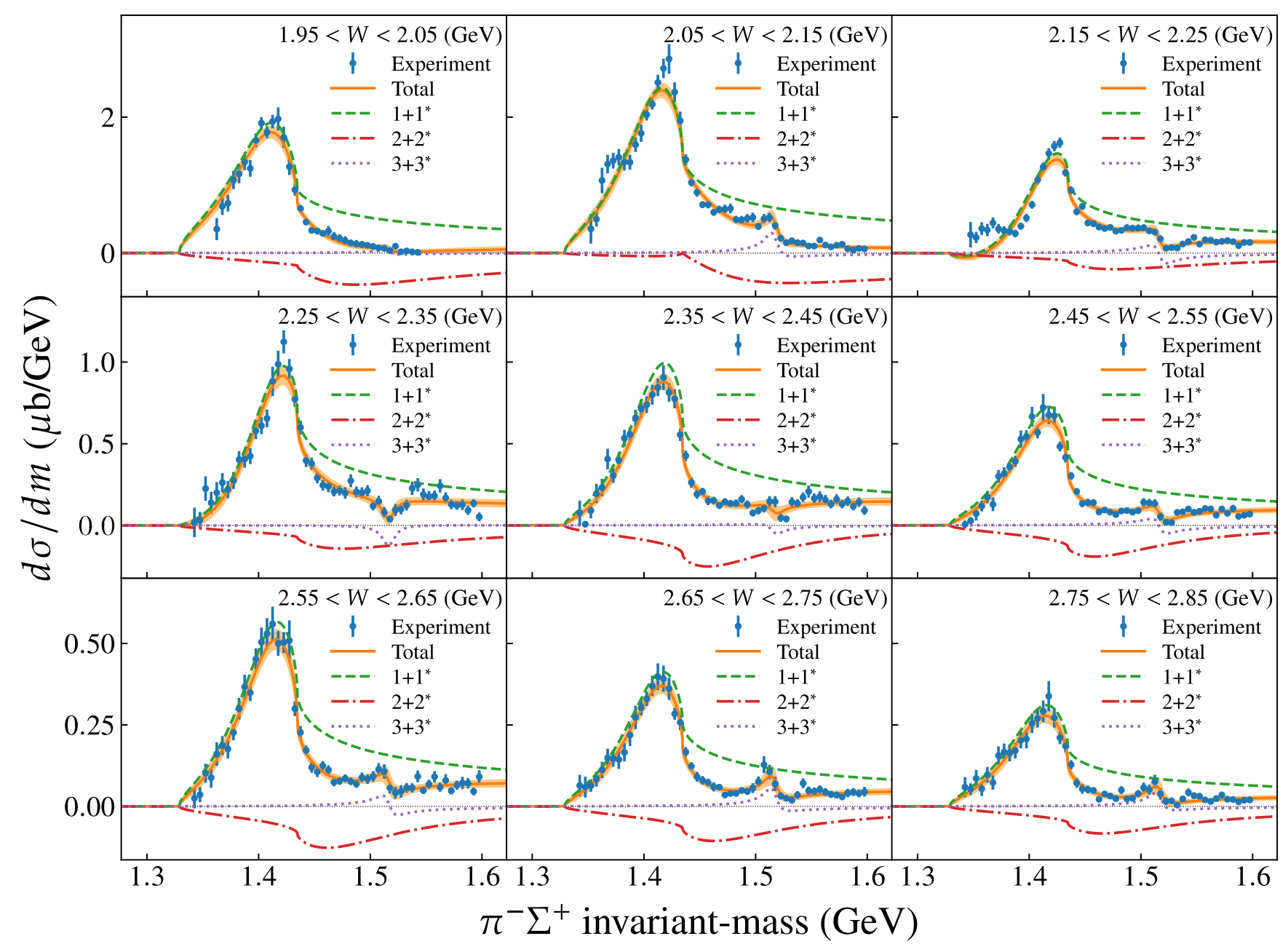

FIG. 5. Results for the invariant-mass distributions of $\pi^{-} \Sigma^{+}$. Details are the same as in Fig. 4.

where $k_{1}$ and $k_{2}$ are the momenta in channel 1 and 2, respectively, and $\tilde{k}_{2}$ is defined by $k_{2}=i \tilde{k}_{2}$. Equations (14) and (15) describe the proper threshold behaviors. Therefore, we will always take into account pairs of poles together in the uniformized Mittag-Leffler expansion. It should be noted, however, that the conjugate poles do not affect the structure of resonances well above the lowest threshold, because they are more distant as the energy becomes higher above the lowest threshold.

If a pole is located close to the physical region and sufficiently away from the threshold, its contribution is approximately given by Eq. (1) with a complex residue as

$$
\begin{aligned}
\operatorname{Im} \frac{c_{n}}{z-z_{n}} \approx & \operatorname{Im} \frac{\tilde{c}_{n}}{\sqrt{s}-\sqrt{s}_{n}} \\
= & A \cos \theta \frac{\Gamma_{n} / 2}{\left(\sqrt{s}-\epsilon_{n}\right)^{2}+\Gamma_{n}^{2} / 4} \\
& +A \sin \theta \frac{\sqrt{s}-\epsilon_{n}}{\left(\sqrt{s}-\epsilon_{n}\right)^{2}+\Gamma_{n}^{2} / 4},
\end{aligned}
$$

where $\sqrt{s}_{n}=\epsilon_{n}-i \Gamma_{n} / 2$ and $\tilde{c}_{n}=c_{n}[d z / d \sqrt{s}]_{\sqrt{s}=\sqrt{s_{n}}}^{-1}=A e^{i \theta}$ are, respectively, the position and residue of the pole in the parametrization, $\sqrt{s}$, corresponding to $z_{n}$. The standard BreitWigner form corresponds to the particular case of $\theta=0$. Note that the approximation in the first line of Eq. (16) only holds for narrow resonances distant from the threshold. On some local coordinate system, the mapping between $\sqrt{s}$ and $z$ is a conformal map, thus preserving the local geometric structure, meaning when $\left|z-z_{n}\right|$ is small and away from critical points, $z=i$ and $1,1 /\left(z-z_{n}\right) \approx \alpha /\left(\sqrt{s}-\sqrt{s}_{n}\right)$. In the neighborhood of the thresholds, or in the region of large $\Gamma$, the mapping between $\sqrt{s}$ and $z$ is warped significantly, so that the approximation breaks down.

\section{APPLICATION TO THE EXPERIMENTAL SPECTRUM OF $\Lambda(1405)$}

We now apply our method to the experimental spectrum of $\Lambda(1405)$, regarding $\Lambda(1405)$ as a resonance in the coupled $I=0$ two channels, $\pi \Sigma$ and $\bar{K} N$. 


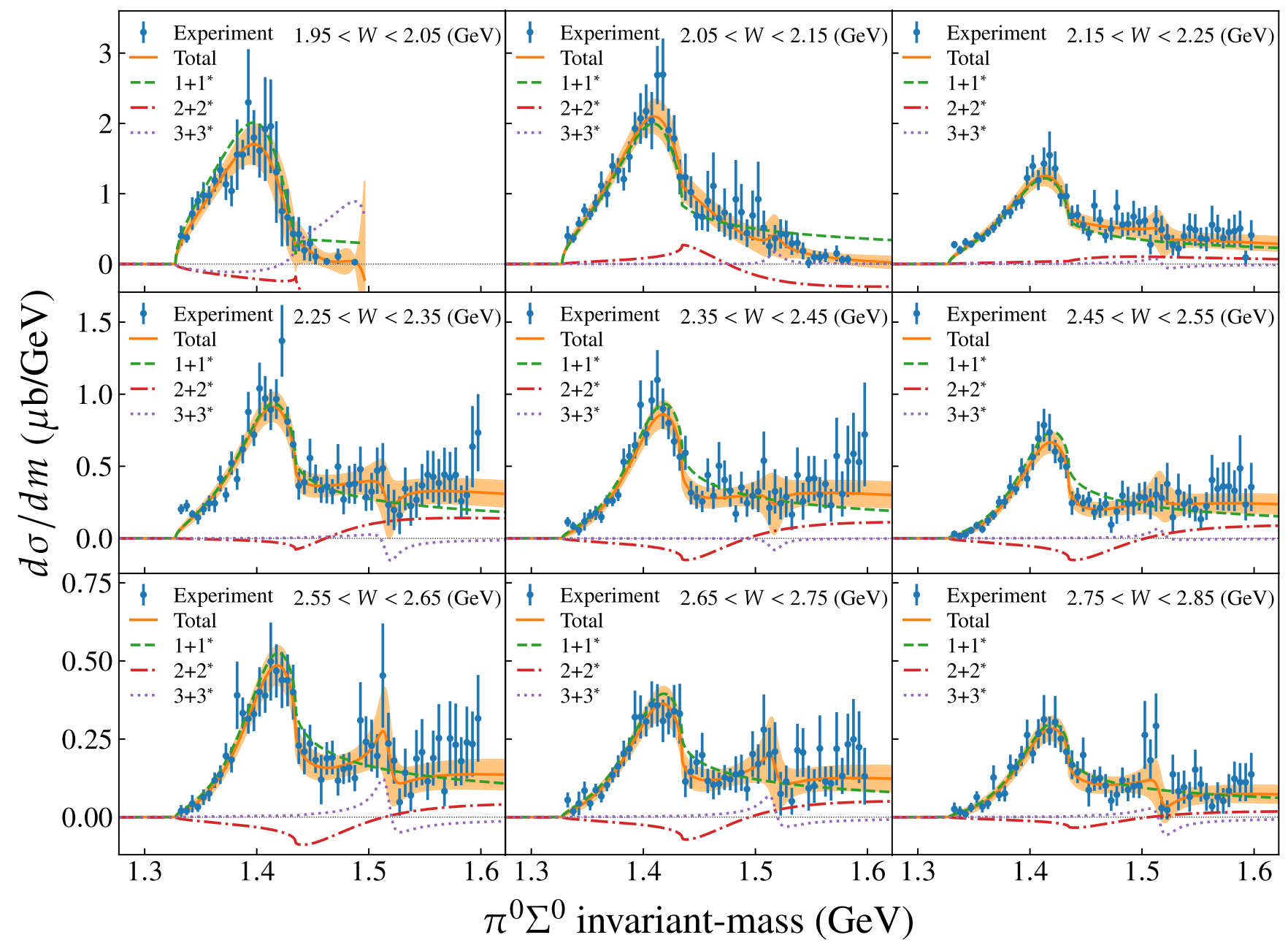

FIG. 6. Results for the invariant-mass distributions of $\pi^{0} \Sigma^{0}$. Details are the same as in Fig. 4. In the $\pi^{0} \Sigma^{0}$ channel the $2 \sigma$-confidence interval is wider than that in the $\pi^{+} \Sigma^{-}$and $\pi^{-} \Sigma^{+}$channels due to large experimental errors.

\section{A. Fitting procedure}

We fit the uniformized Mittag-Leffler expansion with $m$ resonant-pole pairs to the invariant-mass distributions of $\pi^{+} \Sigma^{-}, \pi^{-} \Sigma^{+}$, and $\pi^{0} \Sigma^{0}$ final states in the reaction, $\gamma p \rightarrow$ $K^{+} \pi \Sigma$, measured with CLAS at Jefferson Lab for center-ofmass energies $1.95<W<2.85 \mathrm{GeV}$ [33] as

$$
\frac{d \sigma^{W}}{d M}=\operatorname{Im} \sum_{n=1}^{m}\left(\frac{c_{n}^{W(m)}}{z-z_{n}^{(m)}}-\frac{c_{n}^{W(m) *}}{z+z_{n}^{(m) *}}\right),
$$

and the $K^{-} p$ elastic and inelastic cross sections, $K^{-} p \rightarrow$ $K^{-} p, \bar{K}^{0} n, \pi^{+} \Sigma^{-}, \pi^{-} \Sigma^{+}[26-32]$ as

$$
\sigma^{i f}=\frac{1}{16 \pi^{2} s} \frac{k_{f}}{k_{i}} \operatorname{Im} \sum_{n=1}^{m}\left(\frac{c_{n}^{i f(m)}}{z-z_{n}^{(m)}}-\frac{c_{n}^{i f(m) *}}{z+z_{n}^{(m) *}}\right) .
$$

In Eq. (17), $d \sigma^{W} / d M$ is the distribution of the $\pi \Sigma$ invariant mass $M$, with center-of-mass energy $W$ of the reaction $\gamma p \rightarrow K^{+} \pi \Sigma$. In Eq. (18), $\sigma^{i f}$ is the cross section of the scattering process, $i \rightarrow f, s$ is the center-of-mass energy squared and $k_{i}\left(k_{f}\right)$ is the momentum of the initial (final) state in the center-of-mass frame. The invariant-mass distribution was measured in nine different center-of-mass energies $W$ in the range $1.95-2.85 \mathrm{GeV}$ for each channel, $\pi^{+} \Sigma^{-}, \pi^{-} \Sigma^{+}$, and $\pi^{0} \Sigma^{0}$. Each dataset of $d \sigma^{W} / d M$ and $\sigma^{i f}$ is fit with different residues but common pole positions. Therefore, in the case of $m$ resonant-pole pairs and $N$ data sets we have $m$ and $m N$ complex parameters for the pole positions and residues, respectively. The behavior of the $\pi \Sigma$ invariant-mass distributions in the reaction $\gamma p \rightarrow K^{+} \pi \Sigma$ is sensitive to the $\pi \Sigma$ threshold energies, which are slightly different for the $\pi^{+} \Sigma^{-}$, $\pi^{-} \Sigma^{+}$, and $\pi^{0} \Sigma^{0}$ channels. Therefore, we take into account the difference of the threshold energies with minimum modifications, although we basically regard $\Lambda(1405)$ as a resonance in the coupled two channels, $\pi \Sigma$ and $\bar{K} N$ with isospin as an approximately good quantum number. Namely, we define $z$ differently for each of the $\pi^{+} \Sigma^{-}, \pi^{-} \Sigma^{+}$, and $\pi^{0} \Sigma^{0}$ channels with slightly different $\pi \Sigma$ threshold energies in the fit of the $\pi \Sigma$ invariant-mass distributions. We neither take into account the difference of $\bar{K} N$ threshold energies in the $\pi \Sigma$ invariant-mass distributions nor the difference of $\pi \Sigma$ and $\bar{K} N$ threshold energies in the $K^{-} p$ elastic and inelastic cross sections because it is simply unnecessary. As explained above, each dataset of $d \sigma^{W} / d M$ and $\sigma^{i f}$ is fit with different residues 


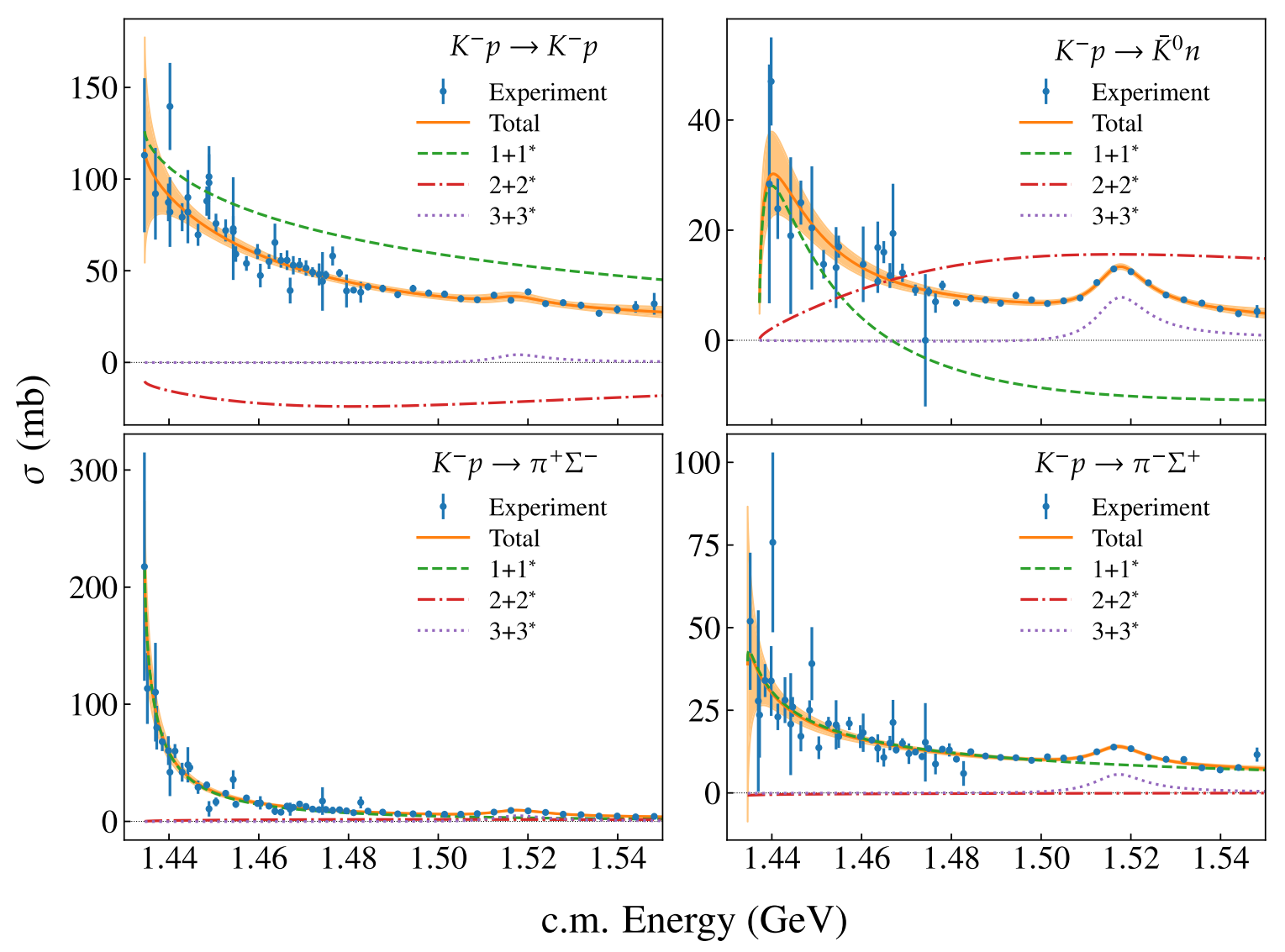

FIG. 7. Results for the cross sections $K^{-} p \rightarrow K^{-} p, \bar{K}^{0} n, \pi^{+} \Sigma^{-}, \pi^{-} \Sigma^{+}$. Details are the same as in Fig. 4.

but common pole positions such that all pole positions are common on the $\sqrt{s}$ plane. This means that the pole positions on the $z$ plane are slightly different for the $\pi^{+} \Sigma^{-}, \pi^{-} \Sigma^{+}$, and $\pi^{0} \Sigma^{0}$ invariant-mass distributions. When we show the pole positions in the $z$ plane, $z$ is defined for the $\pi^{+} \Sigma^{-}$channel. The differences, however, are small and will be ignored in the following discussions.

We start from one resonant-pole pair $m=1$ and gradually increase the number of pairs up to $m=3$. The reduced chisquared values are 5.74, 2.65, and 1.18 for cases, $m=1,2$, and 3 , respectively, and the case $m=3$ best fit the spectrum among them. We present the results of the case $m=3$ in detail in Sec. III B and discuss the convergence of the uniformized Mittag-Leffler expansion from $m=1$ to $m=3$ in Sec. III C.

\section{B. Results of uniformized Mittag-Leffler expansion with $\boldsymbol{m}=\mathbf{3}$}

Figs. 4-6 show the fitted invariant-mass distributions of $\pi^{+} \Sigma^{-}, \pi^{-} \Sigma^{+}, \pi^{0} \Sigma^{0}$ in the reaction $\gamma p \rightarrow K^{+} \pi \Sigma$ and Fig. 7 shows the elastic and inelastic cross sections $K^{-} p \rightarrow K^{-} p$, $\bar{K}^{0} n, \pi^{+} \Sigma^{-}, \pi^{-} \Sigma^{+}$. Since $m=3$ and $N=31$, we have 3 and 93 complex parameters for the pole positions and residues, respectively. The uniformized Mittag-Leffler expansion with $m=3$ fits experimental data very well, which is confirmed also by the reduced chi-squared value 1.18 . The spectrum between the $\pi \Sigma$ and $\bar{K} N$ thresholds is mostly given by the resonant-pole pair $1+1^{*}$, while the spectrum above the $\bar{K} N$

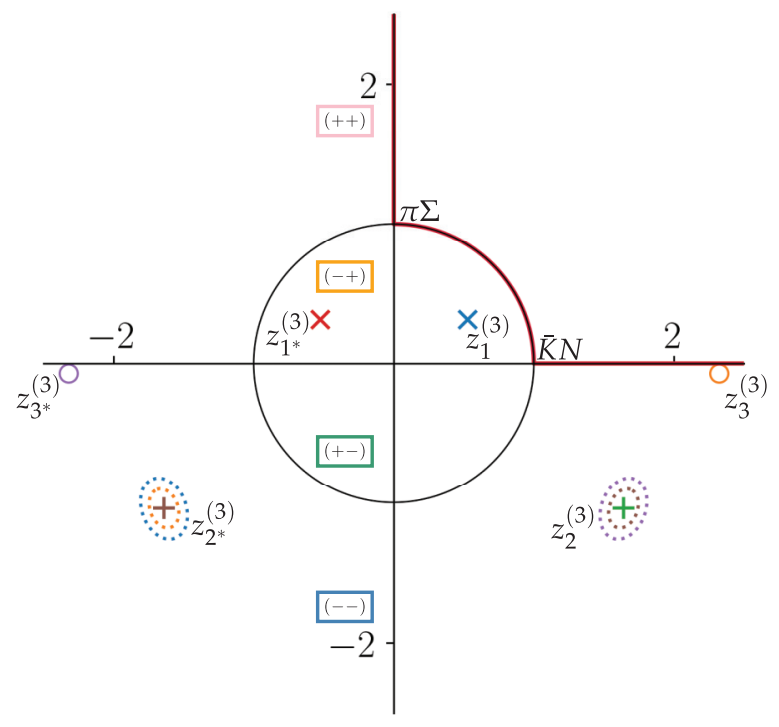

FIG. 8. Results for the pole positions on the $z$ plane by the uniformized Mittag-Leffler expansion with $m=3$. Let us denote $z_{n^{*}}^{(m)}=$ $-z_{n}^{(m) *}$. Two dotted lines around the poles represent the $70 \%$ and $95 \%$ confidence intervals of the position of the poles, respectively, from inside to outside. The (red) thick line represents the physical region accessible in the experiment and the labels $\pi \Sigma$ and $\bar{K} N$ represent the corresponding thresholds. 


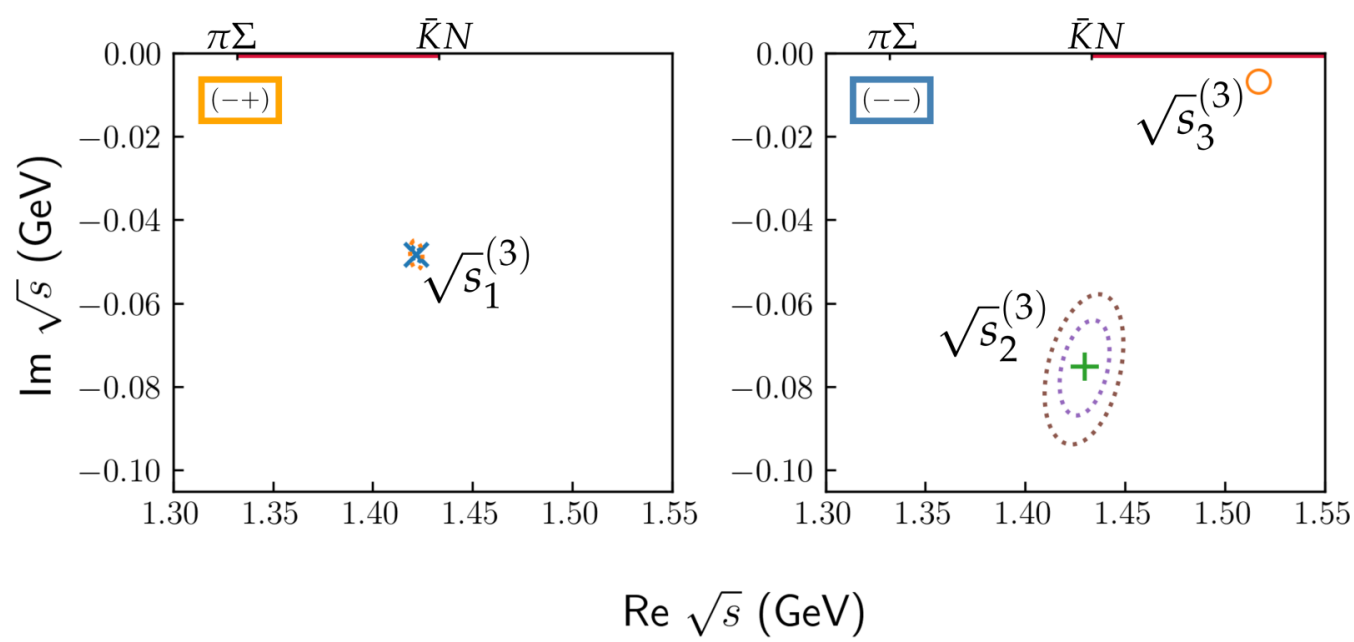

FIG. 9. Results for the pole positions on the $\sqrt{s}$ plane. Details are the same as in Fig. 8.

threshold is given by the sum of $1+1^{*}$ and $2+2^{*}$ except for the narrow structure around $1520 \mathrm{MeV}$, which is explained by the contribution of $3+3^{*}$. The contribution of $3+3^{*}$ can be considered as the remnant of $\Lambda(1520)$, which was not exactly subtracted from the bare experimental data [33]. Also, an extra structure is observed in the invariant-mass distributions of $\pi^{-} \Sigma^{+}$around $1350 \mathrm{MeV}$. To explain such a structure, it may be necessary to consider contributions from additional resonant-pole pairs in the isospin $I=1$ sector.

The positions of poles are tabulated in Table I and are shown on the $z$ plane and $\sqrt{s}$ plane in Figs. 8 and 9, respectively. In Fig. 9, $\left(\operatorname{sgn}\left(\operatorname{Im} q_{1}\right) \operatorname{sgn}\left(\operatorname{Im} q_{2}\right)\right)$ labels the four sheets of the $\sqrt{s}$ plane, where $q_{1}\left(q_{2}\right)$ corresponds to the relative momentum in the $\pi \Sigma(\bar{K} N)$ channel. Sheet $(-+)$ is located adjacent to the physical energy between the $\pi \Sigma$ and $\bar{K} N$ thresholds while sheet $(--)$ is above the $\bar{K} N$ threshold. Pole 1 is positioned on sheet $(-+)$ right below the $\bar{K} N$ threshold at the complex energy of $1420-47 i \mathrm{MeV}$. Poles 2 and 3 are positioned on sheet $(--)$, at the complex energies of $1428-74 i$ and $1514-7 i \mathrm{MeV}$, respectively. Seen only from the perspective of complex energy, pole 2 might seem close to the $\bar{K} N$ threshold, which makes counterintuitive the fact that $2+2^{*}$ mainly contributes to the tail of the spectrum much above the $\bar{K} N$ threshold. Pole 2, however, is not close to the $\bar{K} N$ threshold because it is positioned on Riemann sheet $(--)$, not $(-+)$. In Fig. 8, on the $z$ plane, one can immediately see that the physical domain closest to pole 2 is much above the $\bar{K} N$ threshold.

In Tables II-V, the residues of the poles are presented, which contain the information of wave function and formation processes.

\section{Convergence of uniformized Mittag-Leffler expansion from $m=1$ to $m=3$}

Figure 10 shows a typical invariant-mass distribution of $\pi^{+} \Sigma^{-}$in the reaction $\gamma p \rightarrow K^{+} \pi \Sigma[2.55<W<2.65$ $(\mathrm{GeV})]$ from [33], fit by the uniformized Mittag-Leffler expansion in cases $m=1,2$, and 3. As one can observe, the case $m=1$ fails to reproduce the broad peak structure below the $\bar{K} N$ threshold, whereas the case $m=2$ reproduces most of the spectrum below and above the $\bar{K} N$ threshold. Comparing

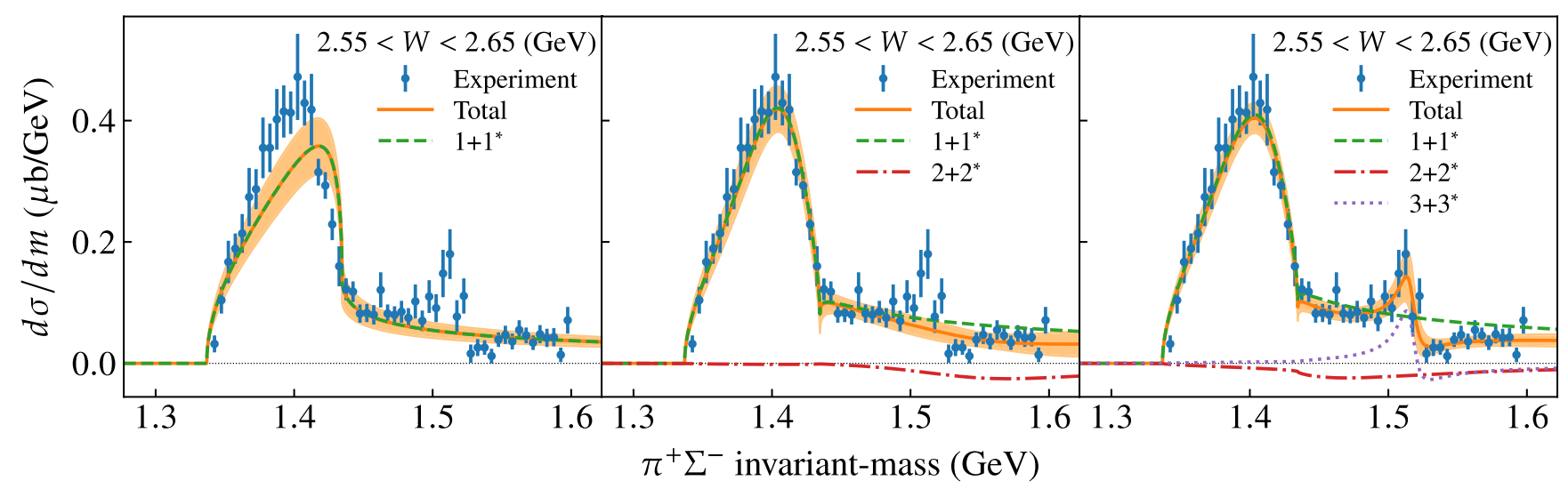

FIG. 10. Results for the invariant-mass distribution of $\pi^{+} \Sigma^{-}$in the reaction $\gamma p \rightarrow K^{+} \pi \Sigma$ by the uniformized Mittag-Leffler expansion with $m=1,2$, and 3 from left to right. 
TABLE II. Results for the residues of the invariant-mass distributions of $\pi^{+} \Sigma^{-}$in units of $\mu \mathrm{b} / \mathrm{GeV}$ in nine bins of center-of-mass energy $W$ by the uniformized Mittag-Leffler expansion with $m=3$.

\begin{tabular}{llll}
\hline \hline$W(\mathrm{GeV})$ & \multicolumn{1}{c}{ Pole 1} & \multicolumn{1}{c}{ Pole 2 } & Pole 3 \\
\hline $1.95-2.05$ & $-0.3486+0.3026 i \pm 0.0154 \pm 0.0149 i$ & $0.2487-0.122 i \pm 0.053 \pm 0.0342 i$ & $-0.0016-0.0029 i \pm 0.0013 \pm 0.0014 i$ \\
$2.05-2.15$ & $-0.3809+0.3245 i \pm 0.0156 \pm 0.0135 i$ & $0.1451-0.1877 i \pm 0.0442 \pm 0.0225 i$ & $-0.0175-0.0081 i \pm 0.0034 \pm 0.0023 i$ \\
$2.15-2.25$ & $-0.2662+0.1989 i \pm 0.0121 \pm 0.0096 i$ & $0.0294-0.0919 i \pm 0.028 \pm 0.0183 i$ & $-0.0108-0.0133 i \pm 0.0029 \pm 0.0021 i$ \\
$2.25-2.35$ & $-0.2539+0.208 i \pm 0.013 \pm 0.0106 i$ & $0.0165-0.0339 i \pm 0.0318 \pm 0.0227 i$ & $0.0014-0.0122 i \pm 0.0023 \pm 0.0021 i$ \\
$2.35-2.45$ & $-0.2016+0.2142 i \pm 0.0131 \pm 0.0104 i$ & $0.0864-0.0442 i \pm 0.0306 \pm 0.0189 i$ & $-0.004-0.0105 i \pm 0.0021 \pm 0.0019 i$ \\
$2.45-2.55$ & $-0.1595+0.1369 i \pm 0.0097 \pm 0.008 i$ & $0.0423-0.0179 i \pm 0.0219 \pm 0.0151 i$ & $-0.0038-0.0091 i \pm 0.0018 \pm 0.0017 i$ \\
$2.55-2.65$ & $-0.1072+0.0925 i \pm 0.008 \pm 0.006 i$ & $0.025-0.0066 i \pm 0.0169 \pm 0.0119 i$ & $-0.0043-0.0065 i \pm 0.0016 \pm 0.0014 i$ \\
$2.65-2.75$ & $-0.0891+0.057 i \pm 0.0065 \pm 0.0046 i$ & $0.0189+0.0133 i \pm 0.0139 \pm 0.01 i$ & $-0.0039-0.0062 i \pm 0.0014 \pm 0.0012 i$ \\
$2.75-2.85$ & $-0.0657+0.0466 i \pm 0.0056 \pm 0.0042 i$ & $0.0161-0.0066 i \pm 0.0115 \pm 0.008 i$ & $-0.0053-0.0051 i \pm 0.0013 \pm 0.0011 i$ \\
\hline
\end{tabular}

TABLE III. Results for the residues of the invariant-mass distributions of $\pi^{-} \Sigma^{+}$in units of $\mu \mathrm{b} / \mathrm{GeV}$ in nine bins of center-of-mass energy $W$ by the uniformized Mittag-Leffler expansion with $m=3$.

\begin{tabular}{llll}
\hline \hline$W(\mathrm{GeV})$ & \multicolumn{1}{c}{ Pole 1} & \multicolumn{1}{c}{ Pole 2} & Pole 3 \\
\hline $1.95-2.05$ & $-0.2247+0.542 i \pm 0.0319 \pm 0.0262 i$ & $0.358-0.2978 i \pm 0.0864 \pm 0.0491 i$ & $-0.0013-0.0038 i \pm 0.0017 \pm 0.0017 i$ \\
$2.05-2.15$ & $-0.1119+0.7353 i \pm 0.035 \pm 0.0301 i$ & $0.0861-0.542 i \pm 0.0823 \pm 0.0456 i$ & $-0.0165-0.0155 i \pm 0.0035 \pm 0.0033 i$ \\
$2.15-2.25$ & $0.1962+0.4702 i \pm 0.02 \pm 0.0162 i$ & $0.2154-0.1012 i \pm 0.0524 \pm 0.0325 i$ & $0.002-0.0171 i \pm 0.0027 \pm 0.0026 i$ \\
$2.25-2.35$ & $0.0662+0.3112 i \pm 0.0144 \pm 0.0129 i$ & $0.1313-0.0568 i \pm 0.0374 \pm 0.0233 i$ & $0.0081+0.001 i \pm 0.0014 \pm 0.002 i$ \\
$2.35-2.45$ & $-0.0017+0.3091 i \pm 0.0116 \pm 0.0116 i$ & $0.2839+0.0335 i \pm 0.0461 \pm 0.0327 i$ & $0.0028-0.0026 i \pm 0.0018 \pm 0.0016 i$ \\
$2.45-2.55$ & $-0.0119+0.2237 i \pm 0.009 \pm 0.0088 i$ & $0.2132+0.017 i \pm 0.0346 \pm 0.0236 i$ & $0.0004-0.006 i \pm 0.0014 \pm 0.0012 i$ \\
$2.55-2.65$ & $-0.0189+0.1726 i \pm 0.0075 \pm 0.0073 i$ & $0.1377-0.0008 i \pm 0.0248 \pm 0.0162 i$ & $-0.0006-0.0038 i \pm 0.001 \pm 0.0011 i$ \\
$2.65-2.75$ & $-0.0123+0.1263 i \pm 0.0062 \pm 0.0055 i$ & $0.1136-0.0044 i \pm 0.02 \pm 0.0131 i$ & $-0.0029-0.0035 i \pm 0.001 \pm 0.0009 i$ \\
$2.75-2.85$ & $-0.0173+0.0932 i \pm 0.0055 \pm 0.005 i$ & $0.0859-0.0121 i \pm 0.016 \pm 0.0096 i$ & $-0.0021-0.0028 i \pm 0.0009 \pm 0.0007 i$ \\
\hline \hline
\end{tabular}

TABLE IV. Results for the residues of the invariant-mass distributions of $\pi^{0} \Sigma^{0}$ in units of $\mu \mathrm{b} / \mathrm{GeV}$ in nine bins of center-of-mass energy $W$ by the uniformized Mittag-Leffler expansion with $m=3$.

\begin{tabular}{lccr}
\hline \hline$W(\mathrm{GeV})$ & Pole 1 & Pole 2 & Pole 3 \\
\hline $1.95-2.05$ & $-0.6515+0.3471 i \pm 0.2256 \pm 0.1211 i$ & $0.5316-1.2492 i \pm 0.7596 \pm 1.3581 i$ & $1.3537-0.6183 i \pm 2.7107 \pm 1.0427 i$ \\
$2.05-2.15$ & $-0.3179+0.5296 i \pm 0.0374 \pm 0.06 i$ & $-0.3174-0.6043 i \pm 0.1764 \pm 0.1197 i$ & $-0.011+0.0019 i \pm 0.0104 \pm 0.0121 i$ \\
$2.15-2.25$ & $-0.1085+0.3535 i \pm 0.0209 \pm 0.0333 i$ & $-0.0763+0.0737 i \pm 0.1051 \pm 0.0997 i$ & $-0.0015-0.009 i \pm 0.0108 \pm 0.0099 i$ \\
$2.25-2.35$ & $-0.053+0.2798 i \pm 0.0154 \pm 0.0245 i$ & $0.0799+0.2387 i \pm 0.0854 \pm 0.0871 i$ & $0.0081-0.0087 i \pm 0.0086 \pm 0.0082 i$ \\
$2.35-2.45$ & $0.0027+0.2895 i \pm 0.0139 \pm 0.0227 i$ & $0.1853+0.2406 i \pm 0.0828 \pm 0.0885 i$ & $0.0052-0.001 i \pm 0.0079 \pm 0.0073 i$ \\
$2.45-2.55$ & $0.0223+0.2323 i \pm 0.0097 \pm 0.0164 i$ & $0.1871+0.2054 i \pm 0.0618 \pm 0.0691 i$ & $-0.0038-0.0032 i \pm 0.0061 \pm 0.0063 i$ \\
$2.55-2.65$ & $0.0088+0.1641 i \pm 0.0084 \pm 0.0141 i$ & $0.1101+0.1044 i \pm 0.0479 \pm 0.0491 i$ & $-0.0051-0.0098 i \pm 0.0054 \pm 0.0042 i$ \\
$2.65-2.75$ & $-0.0018+0.1221 i \pm 0.0076 \pm 0.0126 i$ & $0.0883+0.1107 i \pm 0.0414 \pm 0.0428 i$ & $-0.0026-0.0058 i \pm 0.0047 \pm 0.0038 i$ \\
$2.75-2.85$ & $0.0089+0.094 i \pm 0.0058 \pm 0.009 i$ & $0.0417+0.0439 i \pm 0.0317 \pm 0.0294 i$ & $0.0018-0.0052 i \pm 0.0025 \pm 0.0032 i$ \\
\hline \hline
\end{tabular}

TABLE V. Results for the residues of the cross sections $K^{-} p \rightarrow K^{-} p, \bar{K}^{0} n, \pi^{+} \Sigma^{-}, \pi^{-} \Sigma^{+}$in units $\mu \mathrm{b} / \mathrm{GeV}^{2}$ by the uniformized MittagLeffler expansion with $m=3$.

\begin{tabular}{llll}
\hline \hline & \multicolumn{1}{c}{ Pole 1 } & \multicolumn{1}{c}{ Pole 2 } & Pole 3 \\
\hline$K^{-} p \rightarrow K^{-} p$ & $-5579+21810 i \pm 28869 \pm 8950 i$ & $6572-5272 i \pm 6311 \pm 4234 i$ & $-99.33+32.89 i \pm 39.09 \pm 44.88 i$ \\
$K^{-} p \rightarrow \bar{K}^{0} n$ & $76090-9251 i \pm 25010 \pm 6306 i$ & $-1596+6936 i \pm 3447 \pm 3629 i$ & $-188.2+64.44 i \pm 13.5 \pm 15.32 i$ \\
$K^{-} p \rightarrow \pi^{+} \Sigma^{-}$ & $18960-96.75 i \pm 6251 \pm 1890 i$ & $-125.0+677.6 i \pm 1223.3 \pm 936.0 i$ & $-105.7+35.68 i \pm 7.2 \pm 8.39 i$ \\
$K^{-} p \rightarrow \pi^{-} \Sigma^{+}$ & $-4998+3449 i \pm 5546 \pm 1850 i$ & $82.03+26.41 i \pm 1316.93 \pm 837.59 i$ & $-120.9+26.07 i \pm 8.7 \pm 9.86 i$ \\
\hline \hline
\end{tabular}


TABLE VI. Results for the pole positions by the uniformized Mittag-Leffler expansion with $m=1,2$, and $3 . z_{n}(n=1,2,3)$ is the dimensionless pole position on the $z$ plane and $\sqrt{s}_{n}(n=1,2,3)$ is the pole position on the $\sqrt{s}$ plane with units of GeV.

\begin{tabular}{llll}
\hline \hline & \multicolumn{1}{c}{$m=1$} & \multicolumn{1}{c}{$m=2$} & $m=3$ \\
\hline$z_{1}^{(m)}$ & $0.52+0.012 i \pm 0.01 \pm 0.009 i$ & $0.551+0.323 i \pm 0.007 \pm 0.008 i$ & $0.524+0.316 i \pm 0.006 \pm 0.006 i$ \\
$\sqrt{s}_{1}^{(m)}$ & $1.478-0.003 i \pm 0.004 \pm 0.002 i$ & $1.420-0.042 i \pm 0.001 \pm 0.002 i$ & $1.420-0.048 i \pm 0.001 \pm 0.002 i$ \\
$z_{2}^{(m)}$ & & $2.62-0.75 i \pm 0.09 \pm 0.06 i$ & $1.64-1.04 i \pm 0.07 \pm 0.09 i$ \\
$\sqrt{s}_{2}^{(m)}$ & & $1.53-0.083 i \pm 0.01 \pm 0.004 i$ & $1.43-0.074 i \pm 0.01 \pm 0.004 i$ \\
$z_{3}^{(m)}$ & & & $2.323-0.069 i \pm 0.003 \pm 0.003 i$ \\
$\sqrt{s}_{3}^{(m)}$ & & & $1.5138-0.0068 i \pm 0.0003 \pm 0.0003 i$ \\
\hline \hline
\end{tabular}

the cases $m=1$ and 2, it is clear that we need at least two resonant-pole pair contributions to successfully reproduce the broad peak structure below the $\bar{K} N$ threshold and the continuous spectrum above the $\bar{K} N$ threshold. By the addition of the third resonant-pole pair contribution, the narrow peak structure around $1520 \mathrm{MeV}$ can also be taken into account, resulting in a satisfying approximation of the actual spectrum.

Table VI and Fig. 11 display the fit pole positions for cases, $m=1,2$, and 3 . The position of pole 1 significantly shifts as we increase the number of terms from $m=1$ to $m=2$, whereas it hardly moves when increasing from $m=2$ to $m=3$. This implies that the convergence of pole 1 is almost realized for the case $m=3$. The convergence of pole 2 cannot be seen up to $m=3$ but pole 2 and pole 3 are positioned further and further away from pole 1 .

These behaviors imply that the expansion with $m=3$ is almost convergent in the vicinity of pole 1 .

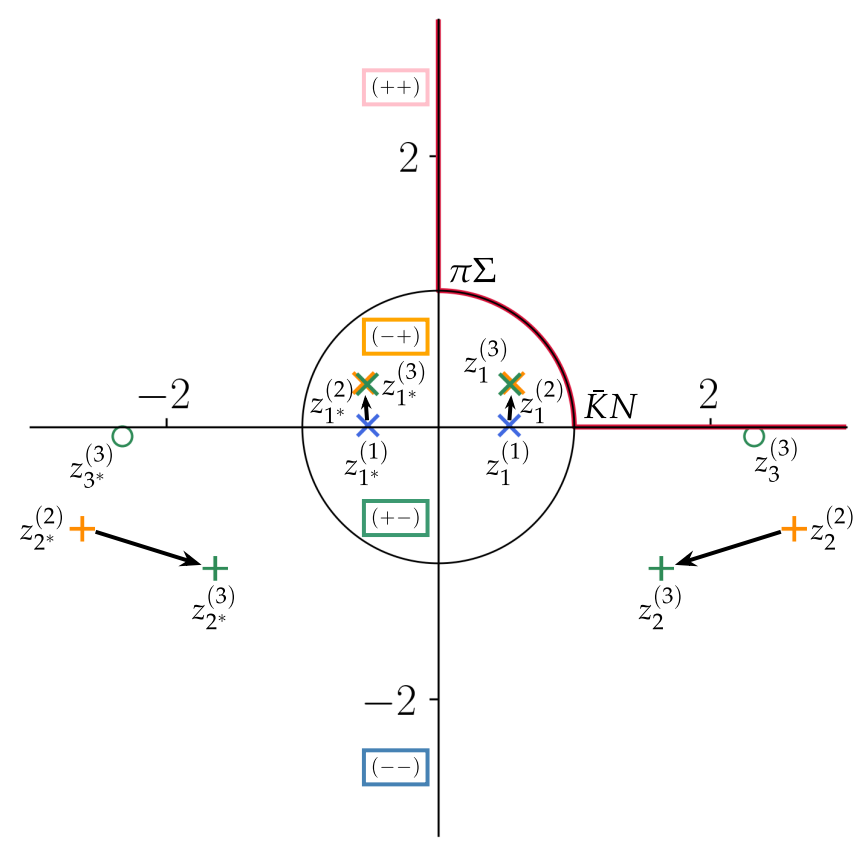

FIG. 11. Results for the pole positions on the $z$ plane by the uniformized Mittag-Leffler expansion with $m=1,2$, and 3.

\section{Discussion}

As stated above, we found only a single pair of poles, $1+1^{*}$, on the $(-+)$ sheet of the complex $\sqrt{s}$ plane, which sufficiently explains the broad peak structure between the $\pi \Sigma$ and $\bar{K} N$ thresholds. Its contribution to the uniformized Mittag-Leffler expansion converges up to $m=3$. This leads us to identify pole 1 as $\Lambda(1405)$. Also, it is natural to identify pole 3 as $\Lambda(1520)$ due to its small width, even though the convergence of $3+3^{*}$ has not been confirmed up to $m=3$. The interpretation of pole 2 is less intuitive, which cannot be identified with any physical resonance. The contribution of $2+2^{*}$ gives the continuous spectrum above the $\bar{K} N$ threshold together with the tail of the contribution of $1+1^{*}$. It should be also noted that the contribution of $2+2^{*}$ is mostly negative.

Usually, the observed spectrum is naively interpreted as the sum of physical resonances and background contributions. However, there is no well-defined criterion when a pole should be identified as a physical resonance or not. In the uniformized Mittag-Leffler expansion, the observed spectrum is represented as a sum of pole contributions, which is well defined. There is no need to identify a pole as a physical resonance or not. Obviously, all the pole contributions in the uniformized Mittag-Leffler expansion cannot be interpreted as resonance contributions in the usual sense.

The results obtained in a model-independent manner by the use of the uniformized Mittag-Leffler expansion support a single-pole picture of $\Lambda(1405)$. To solidify this claim, it may be useful to take into account more than three resonant-pole pair terms in the uniformized Mittag-Leffler expansion.

\section{SUMMARY AND CONCLUSION}

In this paper we applied the uniformized Mittag-Leffler expansion, proposed in our previous paper, to the $\Lambda(1405)$ resonance. We expanded the observable as a sum of resonantpole pairs with a variable which expresses the observable to be single valued, and fit it to experimental data of the invariant-mass distribution of $\pi^{+} \Sigma^{-}, \pi^{-} \Sigma^{+}, \pi^{0} \Sigma^{0}$ final states in the reaction $\gamma p \rightarrow K^{+} \pi \Sigma$ and the elastic and inelastic cross sections $K^{-} p \rightarrow K^{-} p, \bar{K}^{0} n, \pi^{+} \Sigma^{-}, \pi^{-} \Sigma^{+}$. Thus, we determined the resonant energy, width, and residues in a model-independent manner. 
We started from one pair and gradually increased the number of pairs up to three. We observed that the first pair converges while the second and third pairs emerge further and further away from the first pair, which implies that the uniformized Mittag-Leffler expansion with three pairs is almost convergent in the vicinity of $\Lambda(1405)$. The reduced chi-squared values are 5.74, 2.65, and 1.18 for the number of pairs, one, two, and three, respectively, and the uniformized Mittag-Leffler expansion with three pairs satisfactorily fits experimental data. The broad peak structure between the $\pi \Sigma$ and $\bar{K} N$ thresholds regarded to be $\Lambda(1405)$ is explained by the first pair, while the continuous spectrum above the $\bar{K} N$ threshold is given by the first and second pairs except for the narrow structure around $1520 \mathrm{MeV}$, which is explained by the third pair. The results are consistent with the single-pole picture of $\Lambda(1405)$ with a resonant energy of $1420 \pm 1 \mathrm{MeV}$, and a half-width of $48 \pm 2 \mathrm{MeV}$.

In conclusion, the uniformized Mittag-Leffler Expansion approach turns out to be a very powerful and simple method. If experimental data have enough statistics, one can determine the information of near-threshold resonances in a modelindependent way. This is extremely important in order to achieve unbiased understanding of near-threshold resonances.

As an extension of the present work, we can proceed in two directions. One is the application of the present uniformized Mittag-Leffler expansion to other hadron resonances, which are positioned near two two-body thresholds. The other is the extension of the present uniformized Mittag-Leffler expansion to the case with three or more two-body thresholds or cases with three-body thresholds. Both possibilities are presently under our consideration.

\section{ACKNOWLEDGMENTS}

The authors would like to thank Kazuki Yamada for the discussion at the early stage of the work. They would also like to thank Toru Sato, Koichi Yazaki, and the members of the discussion meeting held on the KEK Tokai campus, Yoshinori Akaishi, Akinobu Dote, Toru Harada, Fuminori Sakuma, and Shoji Shinmura.
[1] F.-K. Guo, C. Hanhart, Ulf-G. Meißner, Q. Wang, Q. Zhao, and B.-S. Zou, Rev. Mod. Phys. 90, 015004 (2018).

[2] M. Karliner, J. L. Rosner, and T. Skwarnicki, Annu. Rev. Nucl. Part. Sci. 68, 17 (2018).

[3] G. Breit and E. Wigner, Phys. Rev. 49, 519 (1936).

[4] W. Yamada and O. Morimatsu, Phys. Rev. C 102, 055201 (2020).

[5] R. G. Newton, Scattering Theory of Waves and Particles (Springer, New York, 1982).

[6] M. Kato, Ann. Phys. (NY) 31, 130 (1965).

[7] J. Humblet and L. Rosenfeld, Nucl. Phys. 26, 529 (1961).

[8] W. Romo, Nucl. Phys. A 302, 61 (1978).

[9] J. Bang, F. Gareev, M. Gizzatkulov, and S. Goncharov, Nucl. Phys. A 309, 381 (1978).

[10] T. Berggren, Nucl. Phys. A 389, 261 (1982).

[11] R. Dalitz and S. Tuan, Phys. Rev. Lett. 2, 425 (1959).

[12] R. Dalitz and S. Tuan, Ann. Phys. (NY) 8, 100 (1959).

[13] M. Mai, Few-Body Syst. 59, 61 (2018).

[14] Y. Kamiya, K. Miyahara, S. Ohnishi, Y. Ikeda, T. Hyodo, E. Oset, and W. Weise, Nucl. Phys. A 954, 41 (2016).

[15] A. Cieply, M. Mai, U.-G. Meißner, and J. Smejkal, Nucl. Phys. A 954, 17 (2016).

[16] D. Jido, J. Oller, E. Oset, A. Ramos, and U.-G. Meißner, Nucl. Phys. A 725, 181 (2003).

[17] J. Oller and U. G. Meißner, Phys. Lett. B 500, 263 (2001).

[18] T. Hyodo and W. Weise, Phys. Rev. C 77, 035204 (2008).

[19] T. Hyodo and D. Jido, Prog. Part. Nucl. Phys. 67, 55 (2012).

[20] Y. Ikeda, T. Hyodo, and W. Weise, Phys. Lett. B 706, 63 (2011).
[21] Y. Ikeda, T. Hyodo, and W. Weise, Nucl. Phys. A 881, 98 (2012).

[22] Y. Akaishi, T. Yamazaki, M. Obu, and M. Wada, Nucl. Phys. A 835, 67 (2010).

[23] K. S. Myint, Y. Akaishi, M. Hassanvand, and T. Yamazaki, Prog. Theor. Exp. Phys. 2018, 073 D01 (2018).

[24] J. Révai, Few-Body Syst. 59, 49 (2018).

[25] J. Révai, Few-Body Syst. 61, 32 (2020).

[26] G. S. Abrams and B. Sechi-Zorn, Phys. Rev. 139, B454 (1965).

[27] R. O. Bangerter, M. Alston-Garnjost, A. Barbaro-Galtieri, T. S. Mast, F. T. Solmitz, and R. D. Tripp, Phys. Rev. D 23, 1484 (1981).

[28] J. Ciborowski et al., J. Phys. G: Nucl. Phys. 8, 13 (1982).

[29] M. Csejthey-Barth et al., Phys. Lett. 16, 89 (1965).

[30] W. E. Humphrey and R. R. Ross, Phys. Rev. 127, 1305 (1962).

[31] T. S. Mast, M. Alston-Garnjost, R. O. Bangerter, A. S. BarbaroGaltieri, F. T. Solmitz, and R. D. Tripp, Phys. Rev. D 14, 13 (1976).

[32] M. Sakitt, T. B. Day, R. G. Glasser, N. Seeman, J. Friedman, W. E. Humphrey, and R. R. Ross, Phys. Rev. 139, B719 (1965).

[33] K. Moriya et al. (CLAS Collaboration), Phys. Rev. C 87, 035206 (2013).

[34] J. D. Bjorken and S. D. Drell, Relativistic Quantum Fields (McGraw-Hill, New York, 1965).

[35] A. L. Fetter and J. D. Walecka, Quantum Theory of ManyParticle Systems (McGraw-Hill, New York, 1971).

[36] G. Bertsch and S. Tsai, Phys. Rep. 18, 125 (1975).

[37] O. Morimatsu and K. Yazaki, Prog. Part. Nucl. Phys. 33, 679 (1994). 